\title{
Plasma wall interaction and its implication in an all tungsten divertor tokamak
}

R. Neu, M. Balden, V. Bobkov, R. Dux, O. Gruber, A. Herrmann, A. Kallenbach, M. Kaufmann, C.F. Maggi, H. Maier, H.W. Müller, T. Pütterich, R. Pugno, V. Rohde, A.C.C. Sips, J. Stober, W. Suttrop, C. Angioni, C.V. Atanasiu ${ }^{1}$, W. Becker, K. Behler, K. Behringer, A. Bergmann, T. Bertoncelli, R. Bilato, A. Bottino, M. Brambilla, F. Braun, A. Buhler, A. Chankin, G. Conway, D.P. Coster, P. de Marné, S. Dietrich, K. Dimova, R. Drube, T. Eich, K. Engelhardt, H.-U. Fahrbach, U. Fantz, L. Fattorini ${ }^{2}$, J. Fink, R. Fischer, A. Flaws, P. Franzen, J.C. Fuchs, K. Gál $^{3}$, M. García Muñoz, M. Gemisic-Adamov, L. Giannone, S. Gori, S. da Graca ${ }^{2}$, H. Greuner, A. Gude, S. Günter, G. Haas, J. Harhausen, B. Heinemann, N. Hicks, J. Hobirk, D. Holtum, C. Hopf, L. Horton, M. Huart, V. Igochine, S. Kálvin³ ${ }^{3}$ O. Kardaun, M. Kick, G. Kocsis ${ }^{3}$, H. Kollotzek, C. Konz, K. Krieger, T. Kurki-Suonio ${ }^{4}$, B. Kurzan, K. Lackner, P.T. Lang, P. Lauber, M. Laux, J. Likonen ${ }^{4}$, L. Liu, A. Lohs, K. Mank, A. Manini, M.-E. Manso ${ }^{2}$, M. Maraschek, P. Martin ${ }^{5}$, Y. Martin ${ }^{6}$, M. Mayer, P. McCarthy ${ }^{7}$, K. McCormick, H. Meister, F. Meo ${ }^{8}$, P. Merkel, R. Merkel, V. Mertens, F. Merz, H. Meyer ${ }^{9}$, M. Mlynek, F. Monaco, H. Murmann, G. Neu, J. Neuhauser, B. Nold ${ }^{10}$, J.-M. Noterdaeme, G. Pautasso, G. Pereverzev, E. Poli, M. Püschel, G. Raupp, M. Reich, B. Reiter, T. Ribeiro ${ }^{2}$, R. Riedl, J. Roth, M. Rott, F. Ryter, W. Sandmann, J. Santos $^{2}$, K. Sassenberg, A. Scarabosio, G. Schall, J. Schirmer, A. Schmid, W. Schneider, G. Schramm, R. Schrittwieser ${ }^{11}$, W. Schustereder, J. Schweinzer, S. Schweizer, B. Scott, U. Seidel, F. Serra ${ }^{2}$, M. Sertoli, A. Sigalov, A. Silva ${ }^{2}$, E. Speth, A. Stäbler, K.-H. Steuer, E. Strumberger, G. Tardini, C. Tichmann, W. Treutterer, C. Tröster, L. Urso, E. Vainonen-Ahlgren $^{4}$, P. Varela ${ }^{2}$, L. Vermare, D. Wagner, M. Wischmeier, E. Wolfrum, E. Würsching, D. Yadikin, Q. Yu, D. Zasche, T. Zehetbauer, M. Zilker, H. Zohm

\footnotetext{
Max Planck Institut für Plasmaphysik, EURATOM Association, 85748 Garching, Germany,

${ }^{1}$ Institute of Atomic Physics, EURATOM Association-MEdC, Romania,

${ }^{2}$ CFN, EURATOM Association-IST Lisbon, Portugal,

${ }^{3}$ KFKI, EURATOM Association-HAS, Budapest, Hungary,

${ }^{4}$ HUT, EURATOM Association-Tekes, Helsinki, Finland,

${ }^{5}$ Consorzio RFX, EURATOM Association-ENEA, Padova, Italy,

${ }^{6}$ CRPP/EPFL, Switzerland

${ }^{7}$ Physics Dep., University College Cork, Association EURATOM-DCU, Ireland
} 
${ }^{8}$ NL Ris $\varnothing$, EURATOM Association-RIS $\varnothing$, Roskilde, Denmark,

${ }^{9}$ UKAEA Culham, EURATOM Association-UKAEA, United Kingdom,

${ }^{10}$ Institut für Plasmaforschung, Stuttgart University, Germany,

${ }^{11}$ University of Innsbruck, EURATOM Association-AW, Austria,

Abstract. ASDEX Upgrade has recently finished its transition towards an all-W divertor tokamak, by the exchange of the last remaining graphite tiles to W-coated ones. The plasma start-up was performed without prior boronisation. It was found that the large $\mathrm{He}$ content in the plasma, resulting from DC glow discharges for conditioning, leads to a confinement reduction. After the change to D glow for inter-shot conditioning, the He content quickly dropped and, in parallel, the usual H-Mode confinement with $\mathrm{H}$-factors close to one was achieved. After the initial conditioning phase, oxygen concentrations similar to that in previous campaigns with boronisations could be achieved. Despite the removal of all macroscopic carbon sources, no strong change in $\mathrm{C}$ influxes and $\mathrm{C}$ content could be observed so far. The $\mathrm{W}$ concentrations are similar to the ones measured previously in discharges with old boronisation and only partial coverage of the surfaces with $\mathrm{W}$. Concomitantly it is found that although the $\mathrm{W}$ erosion flux in the divertor is larger than the $\mathrm{W}$ sources in the main chamber in most of the scenarios, it plays only a minor role for the $\mathrm{W}$ content in the main plasma. For large antenna distances and strong gas puffing, ICRH power coupling could be optimized to reduce the $\mathrm{W}$ influxes. This allowed a similar increase of stored energy as yielded with comparable beam power. However, a strong increase of radiated power and a loss of H-Mode was observed for conditions with high temperature edge plasma close to the antennae. The use of ECRH allowed keeping the central peaking of the $\mathrm{W}$ concentration low and even phases of improved $\mathrm{H}$-modes have already been achieved.

PACS numbers: 52.40.Hf, 52.25.Vy, 28.52.Fa, 52.55.Rk, 52.55.Fa 


\section{Introduction}

After the decision for the construction of ITER, plasma wall interaction (PWI) issues moved strongly into the focus of magnetic confinement fusion research. The ITER design tries to optimize the performance by using beryllium, carbon and tungsten as armour materials [1]. However, a future reactor cannot rely on low-Z plasma facing components (PFCs) due to the high erosion $[2,3]$ and solutions with complete high- $Z$ armours have to be developed. The European Fusion Research Program tackles these questions with different approaches, mainly through the ITER like wall project at JET, which will test the ITER material mix [4, 5], and the tungsten programme at ASDEX Upgrade, which investigates the PWI and its implications in an all-W divertor tokamak.

Results from the all Mo device ALCATOR C-Mod [6, 7] suggested that sufficiently high confinement could only be achieved transiently after boronisations by suppressing the high$\mathrm{Z}$ source. However, unlike in Alcator C-Mod, H-Mode plasmas with $H_{H} \approx 1$ could be reliably achieved in ASDEX Upgrade even 200 discharges after boronisation [8]. For the 2007 campaign all PFCs have been equipped with W-coated graphite tiles. To investigate in more detail the effect of covering the W surfaces, the start-up of the 2007 campaign was performed without prior boronisation. In this way, information can also be gained about the conditioning procedure related to the oxygen content, which is of large interest for a future Be-free metal device.

In Sec.2 the final preparation of ASDEX Upgrade to an all $\mathrm{W}$ device is briefly described. Section 3 presents the investigations and results from the start-up procedure without prior boronisation and Sec. 4 describes the comprehensive tungsten influx measurements. Finally, further initial results of the plasma operation and ICRH optimization will be given in Sec. 5, and Sec. 6 will conclude the paper and give an outlook on further investigations and technical modifications.

\section{Preparation of an all-W device}

The area of W PFCs has been continuously increased in ASDEX Upgrade since 1999, and PVD (physical vapour deposition) coatings with a thickness of several microns have been used. The thickness was chosen according to prior measurements of the local erosion [8,9] (and reference therein). As the last step to a complete W coverage of the PFCs, the strikepoint area of the lower divertor, the horizontal frames of the ICRF antennae and all protection tiles for diagnostics were exchanged with W-coated tiles. Since at the strikepoint position of the outer divertor the erosion was locally larger than $0.18 \mathrm{~nm} \mathrm{~s}^{-1}$ [10], it was decided to use thicker $(200 \mu \mathrm{m}) \mathrm{W}$ VPS (vacuum plasma spray) coatings there in order to ensure an erosion lifetime of several campaigns. The coatings were tested in the ion beam facility GLADIS at IPP Garching in thermal screening tests with power loads up to $23.5 \mathrm{MWm}^{-2}$ and cyclic loading tests with 200 pulses at $10.5 \mathrm{MWm}^{-2}$ [11]. The inner strikepoint area, as well as the tiles installed in the main chamber have been W PVD coated with a nominal thickness of 4 $\mu \mathrm{m}$. 
Figure 1 shows photos of newly W-coated areas in ASDEX Upgrade. In the left part (Fig. 1a) the strike point and the baffle of the outer lower divertor, equipped with flush mounted Langmuir probes can be seen. The different surface texture of the flat strike point tile and the curved baffle tile is typical for the VPS and the PVD coating techniques, respectively. The Langmuir probes themselves are implemented with $\mathrm{W}$ bulk material in order to avoid the risk of coating failure at these strongly loaded parts. Figure $1 b$ shows an ICRH frame. The new W-coated limiter tiles (top and bottom of the frame) are clearly distinguishable by their brighter appearance. The two striped tiles at the left and the right side of the limiter frame are long-term marker probes with $\mathrm{Al}(2 \mu \mathrm{m}), \mathrm{Ni}(2 \mu \mathrm{m})$, Mo $(1 \mu \mathrm{m})$ and $\mathrm{W}(0.5 \mu \mathrm{m})$ coatings. In order to further minimize the carbon content of the surfaces, as well as to demonstrate

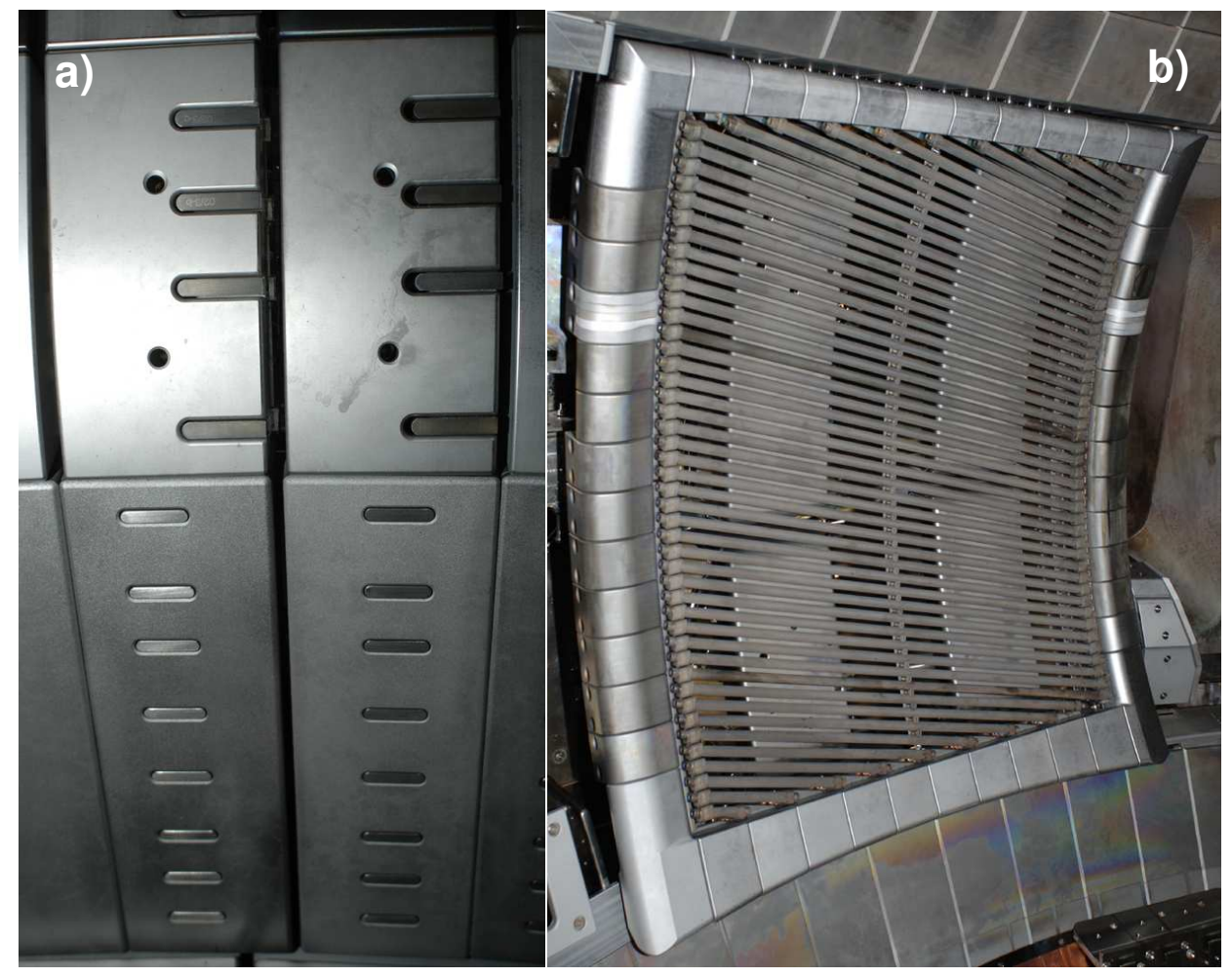

Figure 1. a) A view into the outer divertor of ASDEX Upgrade. The strikepoint tiles are coated with a $200 \mu \mathrm{m}$ VPS W layer, the baffle tiles with a 4 m PVD W layer. The Langmuir probes are manufactured from bulk $W$. b) ICRH antenna with $W$-coated (4 $\mu \mathrm{m} P V D)$ protection limiter. The striped tiles are long-term marker probes with Al (2 $\mu \mathrm{m}), \mathrm{Ni}(2 \mu \mathrm{m}), \mathrm{Mo}(1 \mu \mathrm{m})$ and W (0.5 um) coatings.

operation with bare $\mathrm{W}$ surfaces, the major part of the previously $\mathrm{W}$-coated tiles (inner divertor baffle, central column, cover of the upper passive stabilizer loop) were removed and cleaned mechanically as well as in an ultrasonic bath. Finally, they were baked in atmosphere above $150^{\circ} \mathrm{C}$ for several hours. Additionally, all $\mathrm{W}$ tiles were examined and in case of macroscopic damage of the coating the tile was exchanged with a newly coated one. In order to check the efficiency of the cleaning, sample tiles from the inner divertor baffle - the area with the thickest deposited layers - were investigated with a scanning electron microscope in different 
operational modes. The material composition at the surface was analysed using the energy dispersive x-ray analysis and the voltage of the analysing e-beam $(10 \mathrm{kV})$ was chosen to yield an information depth of a few $\mu \mathrm{m}$ (depending on the layer material and its density). At this voltage only a very weak signal from $\mathrm{W}$ is recorded on the un-cleaned tile, and the dominant species in the surface layer are boron, carbon and oxygen. This observation is in line with Rutherford back-scattering, nuclear reaction analysis and secondary ion mass spectroscopy analyses performed on a similar tile [12]. In contrast, $\mathrm{W}$ is clearly dominating the surface composition on a cleaned $\mathrm{W}$ tile with some contribution of $\mathrm{C}$ and minor amounts of $\mathrm{B}$ and $\mathrm{O}$ in grooves, holes and arc tracks. From these investigations it can be concluded that the cleaning procedure was quite efficient. However, small areas (and probably thin layers) with low-Z content still exist.

\section{Start-up with un-boronised W-PFCs}

ASDEX Upgrade devotes a significant part of its programme to the $\mathrm{W}$ investigations. But there are also several experiments that intentionally use plasma parameters and/or heating scenarios that are not very compatible with W PFCs. For this reason boronisations were regularly performed so far [13] to access and to explore a large working space. Boronisations strongly reduce the impurity level in ASDEX Upgrade. Whereas, oxygen only plays a significant role shortly after a major vent [13], the contents of medium- $Z$ impurities, such as Fe and $\mathrm{Cu}$ that also exist in the plasma, as well as $\mathrm{W}$ are strongly suppressed (1-2 orders of magnitude) after each boronisation.

The reduction of $\mathrm{W}$ influxes and the temporal evolution of the boron coverage are especially evident in ICRH dominated discharges. Similar to observations at Alcator C-Mod $[6,7]$ the effect of boronisation at the areas with strongest erosion (in the case of ASDEX Upgrade these areas seem to be the ICRF limiters) is only very transient and an equilibrium is already reached after 20 discharges. Other areas show longer time constants for recovering the full $\mathrm{W}$ influx but, judging from $\mathrm{W}$ influx and $\mathrm{W}$ concentration measurements, a global equilibrium is reached after about 100 discharges, being less than the typical separation in-between boronisations of about 200 discharges [8]. The observed time constants for B erosion and $\mathrm{W}$ recovery are also consistent with estimates on $\mathrm{B}$ erosion based on measured deuterium fluxes and B layer thicknesses, using B sputtering yields of $5 \times 10^{-4}$ in the divertor and $10^{-2}$ at main chamber PFCs.

However, the fact that confinement was strongly degraded in the un-boronised Alcator C-Mod experiment suggested the question of whether similar behaviour would be found in ASDEX Upgrade with bare $\mathrm{W}$ surfaces. Additionally, it is known that low-Z layers can strongly dominate (see for example $[14,12]$ ) the deuterium inventory and, therefore, a minimisation of such layers would be advantageous for further investigations on D inventories. Motivated by these facts, it was decided to start-up ASDEX Upgrade without boronisation. After pumpdown, the machine was baked at $150^{\circ} \mathrm{C}$ for 10 days, which is twice the duration of the usual procedure. In order to further reduce the absorbed water and nitrogen from the surfaces, several overnight glows in $\mathrm{He}$ (partly with an admixture of $10 \% \mathrm{D}_{2}$ ) were performed. In 
comparison to earlier campaigns it became evident that in the exhaust gas $\mathrm{CO}$ and $\mathrm{CO}_{2}$ were much more prominent, which seems to be typical for a metal device.

Due to a defect of one of the fly-wheel generators during the previous campaign, the power supplies of ASDEX Upgrade had to be re-configured and the voltages on the coils were reduced, leading to a slower response of the plasma control and a smaller available loop voltage. The start-up procedure was slower than usual, because of these technical constraints and a less reliable breakdown and current ramp-up phase compared to boronised conditions. However, after about 20 plasma discharges the current flat-top could be reached and it took only about 5 more discharges to obtain first H-Modes. Figure 2 shows the temporal evolution of the $\mathrm{H} /(\mathrm{H}+\mathrm{D})$ ratio, the divertor He/D influx ratio, the ITER $\mathrm{H}$ factor $H 98$ and the neutron rates during $I_{p}=800 \mathrm{kA}, P_{N B I}=7.5 \mathrm{MW}$ discharges, starting with \#21700 (the recommissioning started around $\# 21600$, but mostly technical trials were performed and only about $100 \mathrm{~s}$ of plasma operation were accomplished in this initial phase). The hydrogen concentration dropped quite rapidly to a value close to $10 \%$, as can be deduced from Fig. 2a). Despite this moderate $\mathrm{H}$ concentration, the confinement remained at $\mathrm{H}$ factors between $0.6-0.8$, although the total radiation in most of these discharges was in the range of $50 \%$. In parallel, an increasing amount of He was observed in the plasma discharges, obviously due to the He overnight glows and the inter-shot He glow discharges ( 5 min duration). The divertor He/D influx ratio, given in Fig. 2b), can also be interpreted as the He concentration in the divertor. Since it is known from experiments and code calculations (see, for example, [15]) that $\mathrm{He}$ is de-enriched in the divertor by a factor of $0.25-0.35$, the He concentrations in the main plasma could have reached values up to $20 \%$, consistent with exploratory CXRS measurements. The storage in and the strong release of He from $\mathrm{W}$ surfaces was already known from the previous campaign and from accompanying laboratory experiments [16], but no strong influence on the confinement was expected. However, after omitting He glow completely and performing 3 minutes of $\mathrm{D}_{2}$ glow only after disruptions, the He concentrations decreased rather quickly and, concomitantly, the confinement factor and the neutron rates (see Fig. 2d)) increased to usual values. Edge density and temperature measurements suggested that a weak edge transport barrier was at least partly responsible for the lower confinement with high He content, and further dedicated experiments will be performed to clarify the underlying physics. Additionally, after the initial conditioning phase it was found, that intershot glow discharges are much less important for plasma ramp-up and density control, than what they have been with graphite PFCs.

In previous campaigns, a strong reduction of the oxygen concentration was observed after each boronisation and a slow increase in-between [13], resulting in an average $\mathrm{O}$ level of a few times $10^{-3}$. Astonishingly, similar levels have been achieved after the initial conditioning phase with the full $\mathrm{W}$ wall without boronisation. Post mortem analysis of the PFCs after the 2006 campaign revealed very low $\mathrm{C}$ deposition at remote areas in the divertor [17], which reflects the strongly reduced primary $\mathrm{C}$ sources consistent with a $\mathrm{C}$ migration and transport model developed to describe the $C$ recycling in the main chamber [18]. Based on these results, a fast reduction of the $\mathrm{C}$ plasma concentrations was expected after removing the last primary sources. However, neither C influx measurements at the central column [19] nor CXR 
spectroscopy show a significant trend yet. Typical values are several $10^{20} \mathrm{~s}^{-1}$ for the gross $\mathrm{C}$-influx and $0.2-1.0 \%$ for the edge $\mathrm{C}$ concentration. These results from spectroscopy are backed up by discharge-resolved measurements with a deposition probe in SOL plasma [20]. The reason for the persisting (low) $\mathrm{C}$ influx and content is not yet clear. However, it has to be stated that only about $800 \mathrm{~s}$ of divertor plasma operation have been performed up to now, and the absolute amount of $\mathrm{C}$ involved is very small.
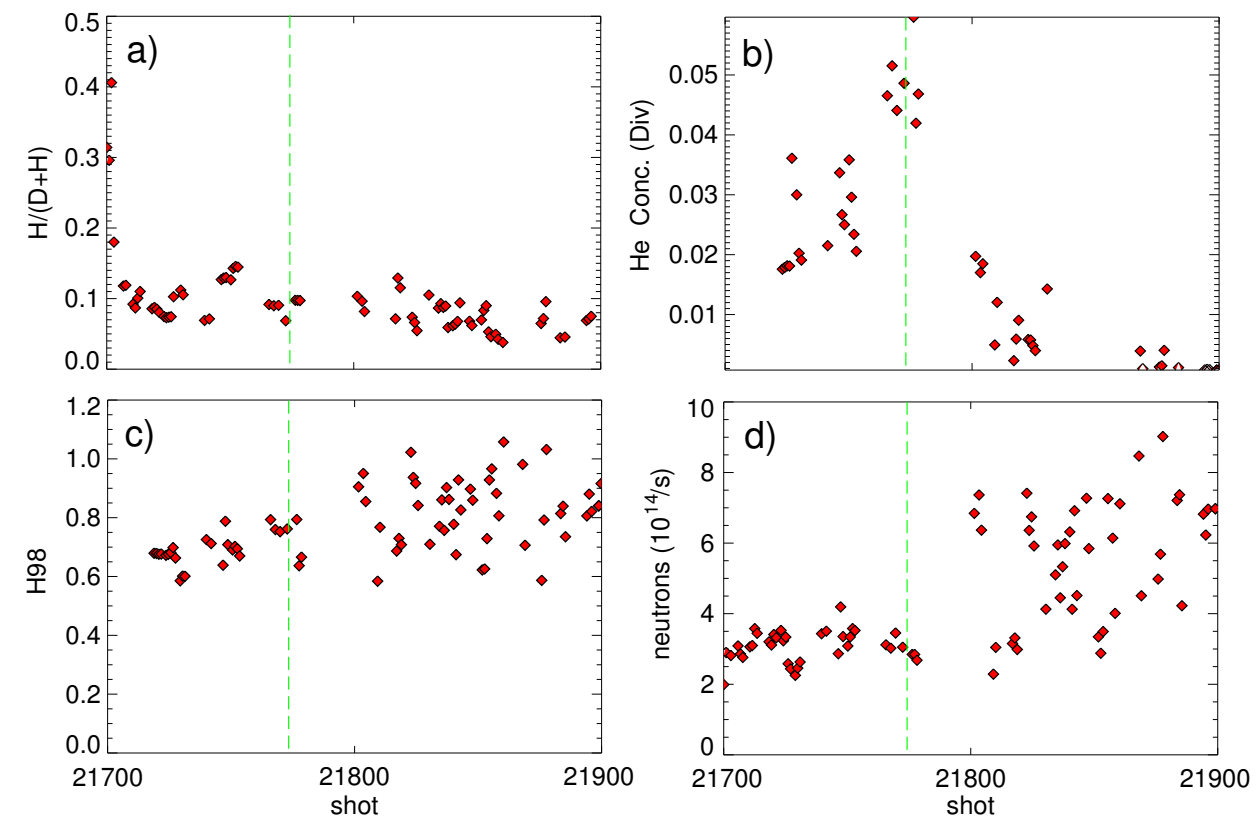

Figure 2. Temporal evolution of the a) $H /(H+D)$ ratio, b) divertor $H e / D$ influx ratio, c) ITER $H$ factor $H 98$ and d) neutron rates for $I_{p}=800 \mathrm{kA}, P_{N B I}=7.5 \mathrm{MW}$ discharges. The vertical dashed lines denote the discharge from which on He-glow was no longer performed.

\section{Tungsten Influxes}

Special emphasis is given to the determination of the tungsten influx in order to elucidate the details of the erosion processes and to assign them to specific plasma scenarios. Therefore, a large set of lines of sight (LOS) have been installed to closely observe the central column, the low field side (LFS) auxiliary and ICRH limiters, as well as the outer lower divertor [21, 19]. The W flux densities are extracted from the W I line at $400.9 \mathrm{~nm}$ using a constant inverse photon efficiency of $S / X B=20$ [22].

Figure 3 shows the temporal evolution of the $\mathrm{W}$ influx profile from the central column in a $I_{p}=800 \mathrm{kA}, \overline{n_{e}} \approx 5 \cdot 10^{19} \mathrm{~m}^{-3}, P_{N B I}=7.5 \mathrm{MW} \mathrm{H}$-mode discharge. The plasma is shifted towards the central column at $t=2.4 \mathrm{~s}$ and again (more slowly) at $t=5 \mathrm{~s}$, characterised by the distance $\Delta R$ (upper part of the figure), which is calculated by mapping the according location along the flux surface to the outer midplane. The radial shift is strongly reflected in the local $\mathrm{W}$ influx. Below the equatorial plane $(z \approx 0 \mathrm{~m})$ the $\mathrm{W}$ influx densities are mostly below the detection limit due to the fact that the upper half of the central column acts as a 
limiter for the particle and power flux crossing the separatrix at the outer midplane. During $t=2.35 \mathrm{~s}-5.00 \mathrm{~s}$, up to $2.1 \mathrm{MW}$ ICRH is launched (see also Fig. 6 for details), leading to an additional increase of the $\mathrm{W}$ flux densities. Interpolation of the measured flux profile along the height of the central column allows the total tungsten source rate to be calculated, assuming toroidal symmetry. For $\Delta R$ above $5 \mathrm{~cm}$, which coincides with the distance where the separatrix is closer to the limiters than to the central column, the total source is below $10^{18} \mathrm{~s}^{-1}$. For smaller distances the source rises to about $1.5 \times 10^{19} \mathrm{~s}^{-1}$ at $\Delta R=2 \mathrm{~cm}$ for pure NBI heating and reaches up to $4 \times 10^{19} \mathrm{~s}^{-1}$ with ICRH [19]. The $\mathrm{W}$ influx from the

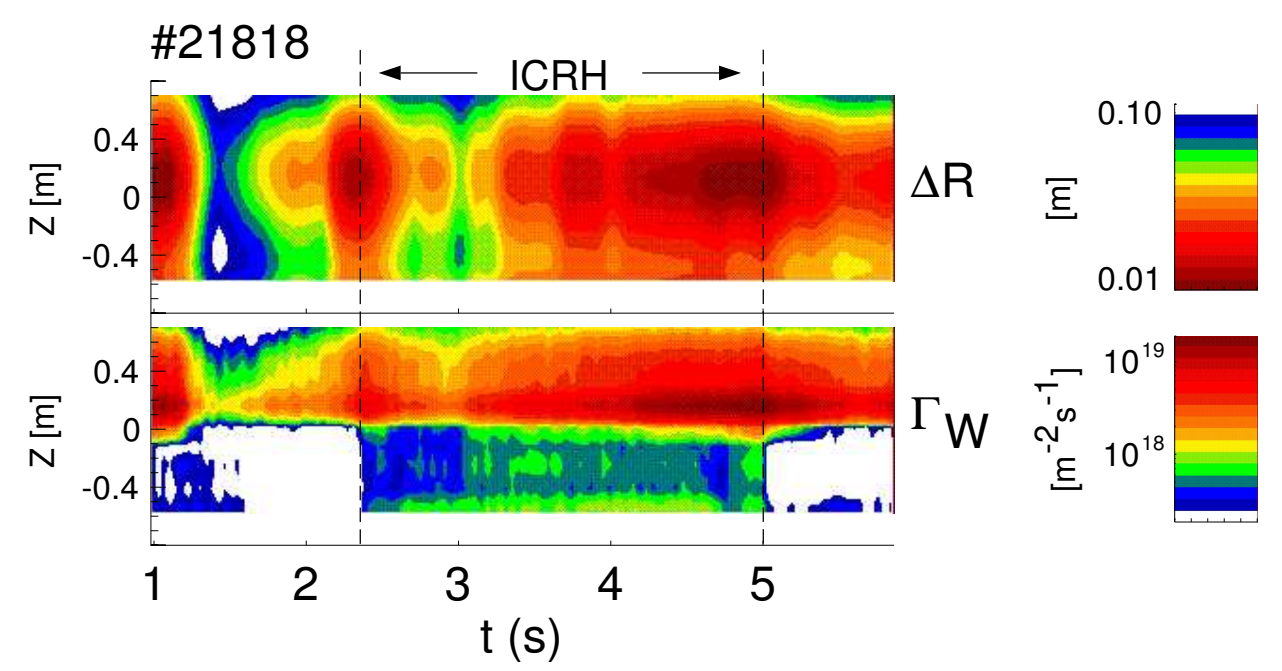

Figure 3. Temporal evolution of the $W$ influx profile from the central column in a $I_{p}=800 \mathrm{kA}$, $\overline{n_{e}} \approx 5 \cdot 10^{19} \mathrm{~m}^{-3}, P_{N B I}=7.5 \mathrm{MW} \mathrm{H}$-mode discharge. The plasma column is shifted towards the central column, characterised by the distance $\Delta R$ (upper part, for details see text) und up to 2.1 MW ICRH is launched from 2.35s - 5.00s (for details see also Fig. 6).

LFS limiters was monitored similarly as in the previous campaigns, resolving the influence of different heating scenarios and the increased sputtering during ELMs [21]. In addition, a set of 5 lines-of-sight positioned, at the same height $z=0.08 \mathrm{~m}$ and separated toroidally by $1.9 \mathrm{~cm}$ at the limiter surface, was installed to measure the toroidal extent of the emission cloud [19]. As in the case of the central column, the distance to the separatrix is the most important parameter which determines the tungsten source at the limiters. Furthermore, a pronounced influence of ICRH is seen, which causes an immediate increase of the influx. The strong $\mathrm{W}$ sputtering is attributed to the acceleration of intrinsic impurities in the rectified parasitic electrical field at the antenna. Extrapolating from the monitored limiters to all 12 LFS limiters, W influxes in the range of $2 \times 10^{18} \mathrm{~s}^{-1}$ (no ICRH) to $7 \times 10^{18} \mathrm{~s}^{-1}$ (with $2 \mathrm{MW}$ ICRH) for rather large distances between separatrix and limiters $(\Delta R>5 \mathrm{~cm})$ are found.

The new $\mathrm{W}$ divertor is monitored by 7 LOS close to the outer strikepoint position and additional 6 LOS on the horizontal tiles at the outer divertor entrance, achieving high spatial resolution and coverage. In addition, the development and construction of spectrometers with a large aperture allows fast, ELM resolved measurements [19]. Figure 6 shows the W flux density (top) and the electron temperature $T_{e}^{d i v}$ (bottom) at the strikepoint of the outer divertor 


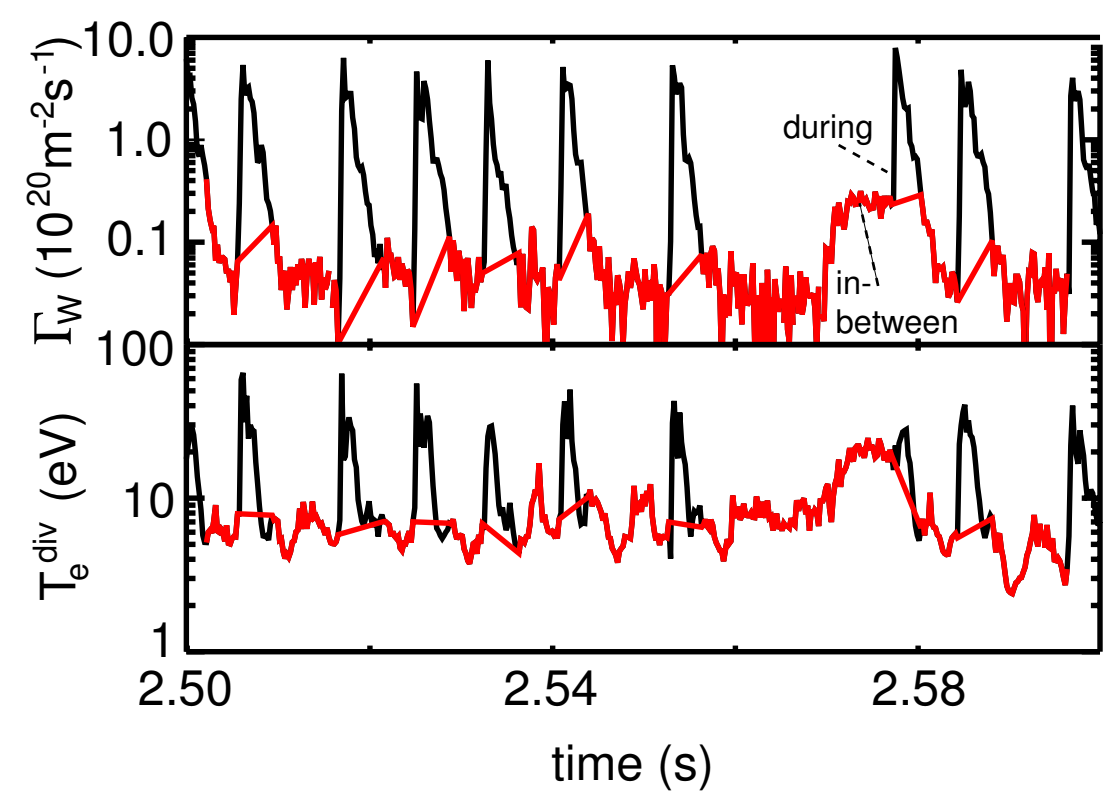

Figure 4. W influx $\Gamma_{W}$ (top) and the electron temperature $T_{e}^{d i v}$ (bottom) at the strikepoint of in the outer divertor in the H-Mode discharge \#21799 with $I_{p}=800 \mathrm{kA}, \overline{n_{e}}=4.9 \cdot 10^{19}$ $m^{-3}, P_{N B I}=7.5 \mathrm{MW}$. The red and black lines distinguish between the values taken in-between ELMS (red) and during ELMS (black) when calculating the ratio of ELM erosion.

in an H-Mode discharge with $I_{p}=800 \mathrm{kA}, \bar{n}_{e}=4.9 \cdot 10^{19} \mathrm{~m}^{-3}, P_{N B I}=7.5 \mathrm{MW}$. Under these conditions, most of the $\mathrm{W}$ erosion $(\approx 90 \%)$ happens during the ELMs, because of the relatively low inter-ELM plasma temperature. If this temperature increases, as for example happens at $t \approx 2.57 \mathrm{~s}$ the inter-ELM erosion increases strongly and accordingly, values down to $50 \%$ for the ratio of in-ELM erosion to the total erosion are observed. Due to the large plasma flux at the strikepoint position the gross $\mathrm{W}$ source in the divertor is larger than the main chamber sources. The values observed up to now in $I_{p}=800 \mathrm{kA}, P_{N B I}=7.5 \mathrm{MW}$ $\mathrm{H}$ mode discharges range from $6 \times 10^{19} \mathrm{~s}^{-1}$ in a high density case to $1.4 \times 10^{20} \mathrm{~s}^{-1}$ at low densities. Despite this fact, no strong influence of the $\mathrm{W}$ divertor source on the $\mathrm{W}$ content in the main plasma is observed. This is in line with earlier investigations comparing the upper $\mathrm{W}$ divertor with the lower graphite divertor [23] and similar investigations at Alcator C-mod [24]. Earlier dedicated experiments with $\mathrm{W}$ injections at the mid-plane and in the divertor [25] elucidate the above result. They revealed a divertor retention of 16 in a medium density $\mathrm{H}$-mode discharge, which means that the amount of $\mathrm{W}$ that reaches the confined plasma is 16 times less when originating from the divertor compared to a similar source at the midplane. 


\section{First operational experiences with the full $W$ wall and influence of ICRH}

Based on the operational experience with W PFCs gathered in earlier campaigns at ASDEX Upgrade, guidelines were set for the current experimental programme. Special care was taken in order not to overload the new $\mathrm{W}$ coatings. As a consequence of the reconfigured power supplies (see Sec. 3) further constraints had to be imposed. Essentially, limits were set for the maximum heating power, the maximum triangularity and the maximum pulse length, depending on the plasma current. Since for the plasma current of $I_{P}=800 \mathrm{kA}$ the limits are rather relaxed $\left(P_{\text {aux }} \leq 10 \mathrm{MW}, t_{\text {disch }} \leq 9 \mathrm{~s}\right)$, most of the program is performed at this current. The toroidal magnetic field (which is not influenced by the defect) was varied from 1.7 $\mathrm{T} \leq B_{t} \leq 2.5 \mathrm{~T}$ with emphasis on the range 2.0-2.5 $\mathrm{T}$ in order to make use of the ECRH and ICRH. The evaluation of $\mathrm{W}$ concentrations was refined to allow the extraction at three temperature ranges spanning from $1 \mathrm{keV}$ up to $5 \mathrm{keV}$ using the $\mathrm{W}$ quasicontinuum and single spectral lines at $5 \mathrm{~nm}$ and the spectral line of Ni-like $\mathrm{W}$ at $0.79 \mathrm{~nm}$ [26].

$\mathrm{H}$-Mode at an intermediate density with an $\mathrm{H}$-factor around 1 was defined as a milestone. It could be achieved already after about $400 \mathrm{~s}$ of plasma operation $\left(\# 21843, I_{P}=800\right.$ $\mathrm{kA}, B_{t}=2.2 \mathrm{~T}, P_{N B I}=7.5 \mathrm{MW}, \bar{n}_{e}=7.5 \cdot 10^{19} \mathrm{~m}^{-3} \approx 0.75 n_{G W}, H 98=1.0 ; n_{G W}$ denotes the Greenwald density), and no significant difference to comparable discharges from last campaign (with boronisations) was apparent. Figure 5 presents the extension of the operational space towards lower densities, where $\overline{n_{e}}=6 \cdot 10^{19} \mathrm{~m}^{-3} \approx 0.55 n_{G W}$ and only a very small feed forward gas puff was imposed. In the first part of the discharge, ECRH is used to keep the peaking of the $\mathrm{W}$ concentration small. This well documented effect $[27,28]$ is nicely seen on the temporal behaviour although only $0.8 \mathrm{MW}$ of ECRH was injected. During this phase the central $\mathrm{W}$ concentration is at the lower end of the range of what is regarded as tolerable for ITER (marked with a green horizontal bar). After the ECRH phase the central $\mathrm{W}$ concentration quickly increases, whereas in the late phase the edge $\mathrm{W}$ concentration even decreases, obviously due to a slow increase of the edge density as can be deduced from the increasing $D_{\alpha}$ baseline. In discharges with central ECRH, slightly adjusted beam timing and without any gas puffing, even phases of improved H-modes $\left(H 98=1.2, \beta_{N}=2.2\right)$ have been achieved [29].

Before the last campaign the ICRH was the usual tool to control the central $\mathrm{W}$ accumulation, because of its high flexibility for operation at different $B_{t}(2.0 \mathrm{~T}-2.7 \mathrm{~T})$. After the $\mathrm{W}$ coating of the poloidal ICRH limiters the beneficial influence on central particle transport from central ICRH could be overridden by the increased $\mathrm{W}$ influx leading to an enhanced $\mathrm{W}$ density over the whole plasma radius. This situation became more pronounced after coating the whole antenna frames and the abdiction of boronisation. Under low density edge conditions a strong increase of $\mathrm{W}$ influx, $\mathrm{W}$ concentration and the total radiation is observed, eventually leading to a back transition to L-Mode, when ICRH is used in a beam heated H-Mode. The only operational regime, found up to now where ICRH is able to significantly increase the plasma energy is the operation with strong gas puffing and an increased gap between separatrix and antennae $(\Delta R=6 \mathrm{~cm}$ instead of $3 \mathrm{~cm})$. This is demonstrated in Fig. 6, where two similar discharges with (righthand side) and without 


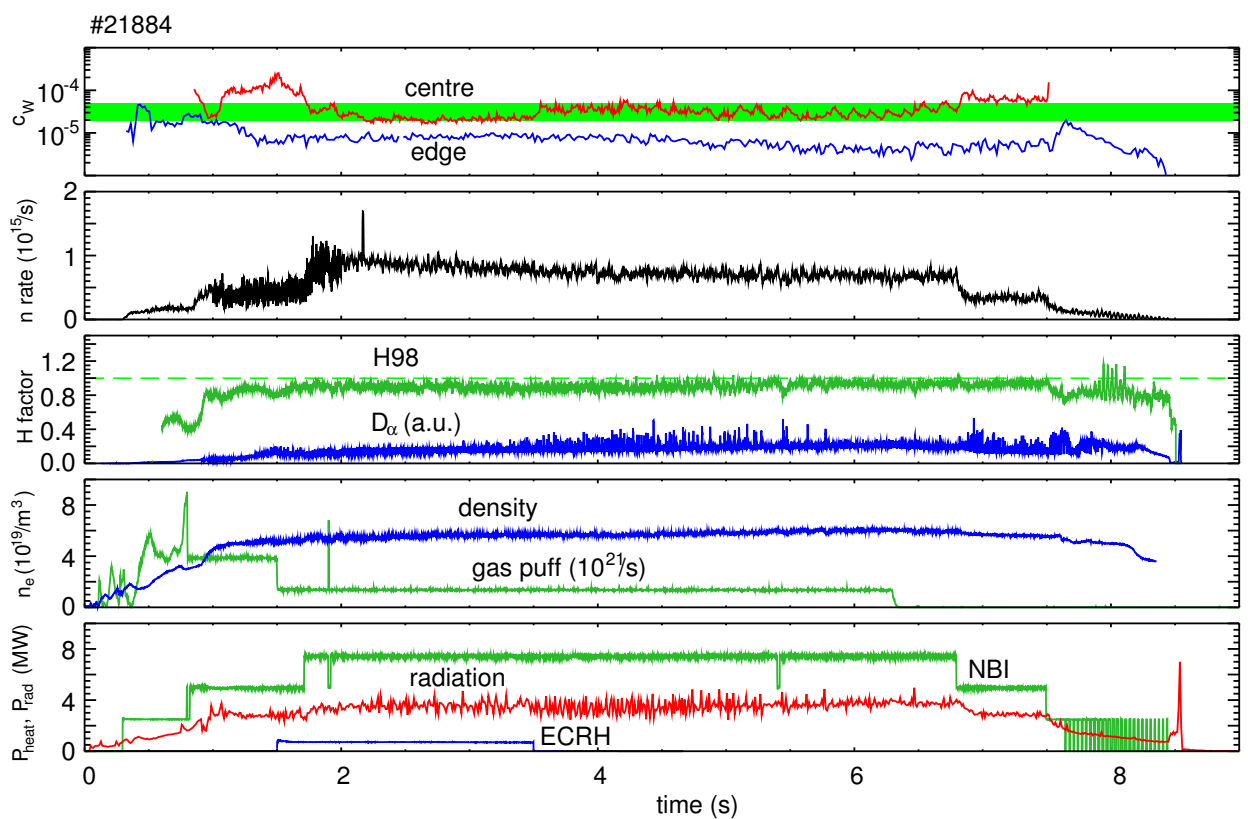

Figure 5. $H$-Mode discharge at $I_{p}=800 \mathrm{kA}, B_{t}=-2.5 \mathrm{~T}$, at low density with a small amount of central ECRH. The parameters shown from top to bottom are: W concentration $\left(c_{W}\right)$ at $\approx 3$ $\mathrm{keV}$ and at $\approx 1 \mathrm{keV}$, neutron rate (n-rate), $H$ factor (H98) and deuterium Balmer $\alpha$ emission $\left(D_{\alpha}\right)$, line averaged density $\left(n_{e}\right)$ and gaspuff-rate, auxiliary heating power $\left(P_{\text {heat }} N B I, E C R H\right)$ and total radiated power $\left(P_{\text {rad }}\right)$. The bar in the top insert marks the extrapolated tolerable central $c_{W}$ in ITER.

(lefthand side) ICRH are compared. As already stated, compared to a discharge without $\mathrm{ICRH}$, the total radiation is significantly larger and the edge density of $\mathrm{W}$ is higher in the discharge with ICRH. At the same time, the $\mathrm{W}$ peaking is smaller, the $\mathrm{H}$-factor increases during ICRH to about 1 and also a significant increase in the neutron rate is observed.

\section{Conclusions and Outlook}

ASDEX Upgrade has recently finished its transition towards an all-W divertor tokamak, by mounting W-coated tiles on all remaining diagnostic covers,

the toroidal ICRH limiters and the divertor strikepoint tiles. The plasma start-up was performed without prior boronisation and no boronisation has been performed up to date. It was found that He from DC glow discharges led to a lower global confinement and after the change to D glow for inter-shot conditioning, the usual $\mathrm{H}$-Mode confinement with $\mathrm{H}$-factors close to one could be achieved. The oxygen concentrations were similar as in previous campaigns with boronisations and despite the removal of all macroscopic carbon sources no strong change in $\mathrm{C}$ influxes and $\mathrm{C}$ content could be observed yet.

Special emphasis has been given to the measurement of all W sources in order to allow for detailed investigation on their influence on the main plasma $\mathrm{W}$ content. The $\mathrm{W}$ divertor source is larger than the integral over the main chamber sources in most of the scenarios, but it plays only a minor role for the $\mathrm{W}$ content in the main plasma. The $\mathrm{W}$ concentrations are similar 


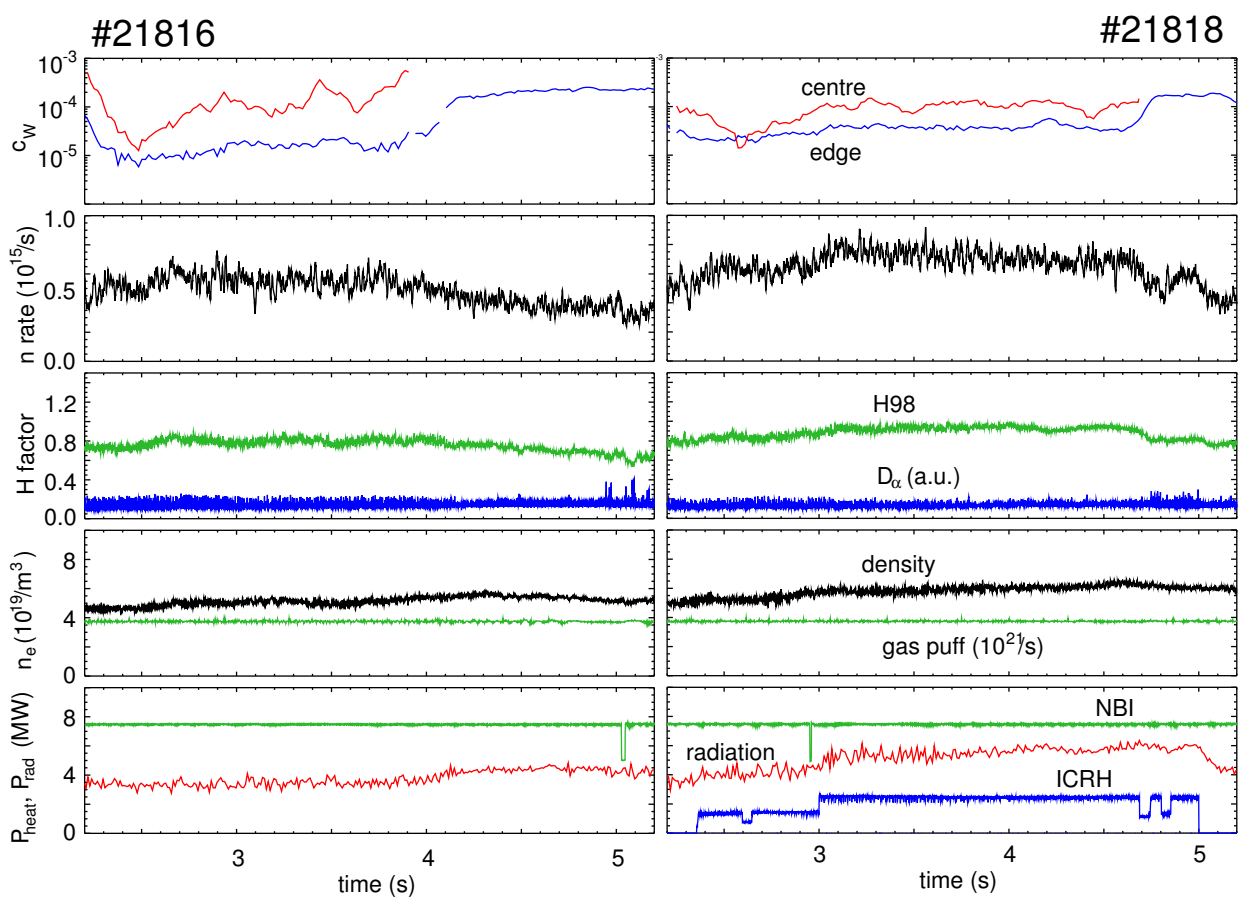

Figure 6. Comparison of similar discharges with and without ICRH $\left(I_{p}=800 \mathrm{kA}, B_{t}=-2.5\right.$ T). The discharge with ICRH is shifted by $1 \mathrm{~cm}$ further away from the outer limiters compared to the one without ICRH. The plasma parameters are denoted similar as in Fig. 5

to the ones found previously in discharges with old boronisation and range from significantly below $10^{-5}$ (flat $c_{W}$ profile, high density edge) up to several $10^{-4}$ (W accumulation), substantiating earlier findings on the effect of boronisation. The $\mathrm{W}$ source during ICRH could be reduced by large wall clearance and strong gas puffing, which allowed a similar increase of stored energy as yielded with comparable beam power. However, a strong increase of radiated power and a loss of H-Mode was observed for conditions with high temperature edge plasmas close to the antennae. The use of ECRH allowed the central peaking of the $\mathrm{W}$ concentration to be kept low similar to what has been reported earlier.

This paper can only give an interim report on the ongoing experiments at the all-W divertor tokamak ASDEX Upgrade. Since the tolerable central $c_{W}$ for ITER is extrapolated to be about $5 \times 10^{-5}$ [30], it is clear that a detailed analysis of the data is needed to supply information in time for decisions concerning plasma wall interaction in ITER. In the remaining as well as in the next campaign strong emphasis will be put on further investigations concerning the elucidation of the role of the different $\mathrm{W}$ sources for the central $\mathrm{W}$ content in order to optimise the performance with all-W PFCs. This is especially true for radiatively cooled scenarios, which will be performed using different gas species or even mixtures of them to substitute carbon, which is expected to gradually disappear. An important task remains the investigation of the operation of ICRH in a high-Z environment to answer the question, whether and under what circumstances both are compatible with each other. In support of this, local gas puffing through an antenna limiter will be commissioned soon and faceplates will be installed at the 
corners of one antenna in order to study the effect of uncompensated electrical fields. Gas balance measurements have been started and will be supported by post mortem analyses of W samples. Avoiding boronisation will offer a unique opportunity to get samples with only small amounts of low-Z deposition. Finally, efforts will be made to extend the operational space with high-Z PFCs to regimes with confinement improvement above the conventional $\mathrm{H}$-modes. The initial results obtained so far, suggest that there might be some room when using ECRH. However, for investigations as close as possible to ITER parameters (high $n_{e}$ and high $T_{e}$ and $T_{i}$ ), the full capabilities of the ASDEX power supplies have to be re-established, which is only foreseen for the end of next year.

\section{References}

[1] R. Aymar, P. Barabaschi, and Y. Shimomura, Plasma Phys. Control. Fusion 44, 519-565 (2002).

[2] G. Janeschitz et al., Nucl. Fusion 40, 1197 - 1221 (2000).

[3] H. Bolt et al., J. Nucl. Mater. 307-311, 43-52 (2002).

[4] J. Pamela, G. Matthews, V. Philipps, and R. Kamendje, J. Nucl. Mater. 363-365, 1 - 11 (2007).

[5] G. Matthews et al., Physica Scripta T128, 137-143 (2007).

[6] B. Lipschultz et al., Physics of Plasmas 13, 056117 (2006).

[7] B. Lipschultz et al., J. Nucl. Mater. 363-365, 1246-1250 (2007).

[8] R. Neu et al., J. Nucl. Mater. 363-365, 52-59 (2007).

[9] R. Neu et al., J. Nucl. Mater. 367-370, 1497 - 1502 (2007).

[10] M. Mayer et al., Physica Scripta T128, 106-111 (2007).

[11] R. Neu et al., Physica Scripta T128, 150-156 (2007).

[12] M. Mayer et al., Nucl. Fusion in press (2007).

[13] V. Rohde et al., J. Nucl. Mater. 363-365, 1469-1374 (2007).

[14] D. Schleußner et al., J. Nucl. Mater. 266-269, 1296-1302 (1999).

[15] H.-S. Bosch et al., Plasma Phys. Control. Fusion, 1771-1792 (1997).

[16] K. Schmid, T. Schwarz-Selinger, W. Jacob, and R. Dux, Nucl. Fusion 47, 984-989 (2007).

[17] M. Mayer et al., to be published (2007).

[18] A. Kallenbach et al., J. Nucl. Mater. 363-365, 60-65 (2007).

[19] R. Dux et al., Impurity Influx in the All Tungsten ASDEX Upgrade, in Europhysics Conference Abstracts (CD-ROM, Proc. of the 34th EPS Conference on Plasma Physics, Warsaw, 2007), pages P-1.052, Geneva, 2007, EPS.

[20] W. Schustereder, A. Herrmann, K. Krieger, V. Rohde, and ASDEX Upgrade Team, Impurity fluxes in the scrape-off layer of ASDEX Upgrade in the full tungsten wall configuration, in Europhysics Conference Abstracts (CD-ROM, Proc. of the 34th EPS Conference on Plasma Physics, Warsaw, 2007), pages P2.034, Geneva, 2007, EPS.

[21] R. Dux et al., J. Nucl. Mater. 363-365, 112-116 (2007).

[22] A. Thoma et al., Plasma Phys. Controlled Fusion 39(9), 1487-1499 (1997).

[23] R. Neu et al., Nucl. Fusion 45(3), 209-218 (2005).

[24] B. Lipschultz et al., Nucl. Fusion 41, 585 - 596 (2001).

[25] A. Geier, H. Maier, R. Neu, K. Krieger, and ASDEX Upgrade Team, Plasma Phys. Controlled Fusion 44(10), 2091-2100 (2002).

[26] T. Pütterich, R. Neu, R. Dux, and ASDEX Upgrade Team, Spectroscopic Diagnostic of Tungsten in Fusion Plasmas, in Europhysics Conference Abstracts (CD-ROM, Proc. of the 34th EPS Conference on Plasma Physics, Warsaw, 2007), pages P-5.103, Geneva, 2007, EPS.

[27] R. Neu et al., Plasma Phys. Controlled Fusion 44(6), 811-826 (2002).

[28] R. Dux et al., Plasma Phys. Controlled Fusion 45(9), 1815-1825 (2003).

[29] J. Stober et al., First experiments with the extended ECRH system on ASDEX Upgrade, in Europhysics 
Conference Abstracts (CD-ROM, Proc. of the 34th EPS Conference on Plasma Physics, Warsaw, 2007), page P5.138, Geneva, 2007, EPS.

[30] A. Kallenbach et al., Plasma Phys. Controlled Fusion 47(12B), B207-B222 (2005). 